\title{
Role of AMPK signalling pathway during compensatory growth in pigs
}

\author{
Maria Ballester ${ }^{{ }^{*}}$ (D), Marcel Amills², Olga González-Rodríguez ${ }^{1}$, Tainã F. Cardoso ${ }^{2}$, Mariam Pascual', \\ Rayner González-Prendes², Núria Panella-Riera³ ${ }^{3}$ Isabel Díaz³, Joan Tibau ${ }^{4}$ and Raquel Quintanilla ${ }^{1 *}$
}

\begin{abstract}
Background: The molecular basis of compensatory growth in monogastric animals has not yet been fully explored. Herewith, in this study we aim to determine changes in the pig skeletal muscle transcriptome profile during compensatory growth following a feed restriction period. A RNA-Seq experiment was performed with a total of 24 females belonging to a Duroc commercial line. Half of the animals received either a restricted (RE) or ad libitum (AL) diet during the first fattening period (60-125 d of age). After that, all gilts were fed ad libitum for a further $\sim 30$ $\mathrm{d}$ until the age of $\sim 155 \mathrm{~d}$, when animals were slaughtered and samples of gluteus medius muscle were harvested to perform RNA-Seq analyses and intramuscular fat content determination.

Results: During the period following food restriction, RE animals re-fed ad libitum displayed compensatory growth, showed better feed conversion rate and tended to deposit more subcutaneous fat than AL fed animals. Animals were slaughtered in the phase of accelerated growth, when RE animals had not completely compensated the performance of AL group, showing lower live and carcass weights. At intramuscular level, RE gilts showed a higher content of polyunsaturated fatty acids during the compensatory growth phase. The comparison of RE and AL expression profiles allowed the identification of 86 ( $\log _{2}$ Fold-Changel $>1, p_{\text {adj }}<0.05$ ) differentially expressed (DE) genes. A functional categorization of these DE genes identified AMPK Signaling as the most significantly enriched canonical pathway. This kinase plays a key role in the maintenance of energy homeostasis as well as in the activation of autophagy. Among the DE genes identified as components of AMPK Signaling pathway, five out of six genes were downregulated in RE pigs.

Conclusions: Animals re-fed after a restriction period exhibited a less oxidative metabolic profile and catabolic processes in muscle than animals fed ad libitum. The downregulation of autophagy observed in the skeletal muscle of pigs undergoing compensatory growth may constitute a mechanism to increase muscle mass thus ensuring an accelerated growth rate. These results reveal that the downregulation of AMPK Signaling plays an important role in compensatory growth in pigs.
\end{abstract}

Keywords: Pig, Feed restriction, Compensatory growth, Skeletal muscle, RNA-Seq, Autophagy, Energy homeostasis, AMPK

\section{Background}

In the pork industry, different feeding strategies have been applied to modify growth performance, carcass composition and meat quality traits (reviewed in $[1,2]$ ). Among these strategies, the benefits of having a compensatory growth (CG) response induced by an energy

\footnotetext{
* Correspondence: maria.ballester@irta.cat; raquel.quintanilla@irta.cat ${ }^{1}$ Animal Breeding and Genetics Programme, Institute for Research and Technology in Food and Agriculture (IRTA), Torre Marimon, E08140 Caldes de Montbui, Spain

Full list of author information is available at the end of the article
}

and/or nutrient intake restriction period followed by subsequent ad libitum feeding have been explored in several studies [2-6]. There is, however, some controversy regarding the possible benefits of feed restriction on final performance. Despite initial decreases in growth rates and fat deposition, the CG phenomenon has been studied for several decades in pigs (reviewed in [7]). The magnitude of compensation (partial, complete or no CG) is affected by multiple factors such as the type, degree, timing and duration of growth restriction, body development at the beginning of the restriction period and

(c) The Author(s). 2018 Open Access This article is distributed under the terms of the Creative Commons Attribution 4.0 International License (http://creativecommons.org/licenses/by/4.0/), which permits unrestricted use, distribution, and 
genotype (reviewed in $[7,8]$ ). As one main benefit, some studies pointed out an improvement in feed efficiency in animals under restriction [2], and in some cases the reduction of back fat could be considered as desirable.

Over the last decades, the amount and composition of fat in muscle have gained special interest in the food industry due to their profound effect on meat quality. Intramuscular fat content and composition directly impact the nutritional quality of food as well as its sensory attributes which play an integral role in the overall consumer acceptance, such as meat tenderness and flavour $[9,10]$. The effect of feed restriction on muscle fat deposition and fatty acid profile should be investigated, especially in systems aimed at obtaining a product of differential quality. To this extent, Heyer and Lebret [11] reported that food restriction did not cause significant changes on intramuscular fat content, whereas Daza et al. [2] observed changes in the muscle fatty acid profile of Iberian pigs under food restriction.

Recently, Keogh et al. [12] have described changes in the bovine skeletal muscle profile of cattle under restriction and during the subsequent early CG using RNA-Seq. These studies indicated that the $\beta$-oxidation of fatty acids, oxidative phosphorylation and the tricarboxylic acids cycle were upregulated in restricted cattle, but the direction of the expression changes reverses during the re-feeding and CG phase [12]. However, the molecular basis of CG in ruminants and monogastrics could be very different. Although different studies have described the physiological aspects associated with feed restriction and CG in several species $[8,13]$, there is a lack of literature analysing gene expression changes associated to CG in pigs. The objective of this study is to gain new insights into the biological and molecular mechanisms underlying CG and phenotypic changes induced by feed restriction in pigs.

\section{Methods}

\section{Animals and experiment}

An experimental device was set up for evaluating changes in the muscle transcriptome of pigs during early CG induced by feed restriction followed by subsequent ad libitum feeding. On the basis of previous observations of CG in conventional pig breeds or crossbreds (reviewed in Lebret [1]), the refeeding period after restriction was established in 30-35 $\mathrm{d}$ to ensure that animals were in the phase of accelerated growth. The experiment was carried out with 24 gilts from a commercial Duroc line which is devoted to produce high quality cured products. These 24 females were born in the same week (25th - 31st January 2015) in 12 different litters, i.e. 12 pairs of full sibs. After weaning at 3-4 weeks of age, female piglets were moved from the farm of origin to the IRTA (Institut de Recerca i Tecnologia Agroalimentàries)-Pig Experimental Farm in Monells (Girona, Spain).
At their arrival, animals were housed in transition devices and fed ad libitum a standard transition diet until approximately 2 months of age (around $18 \mathrm{~kg}$ of live weight). Gilts were then transferred to the fattening pens, where they were housed individually and distributed in two dietary conditions for the first part of the fattening period: fed ad libitum (AL group) and fed under restriction (RE group). Each of the sibling pairs were divided into the two dietary treatments, so that the 12 gilts in one group were full sibs of the 12 gilts in the other. Gilts were housed individually and fed with the same standard grower feed (Additional file 1: Table S1). During the first part of fattening period, between 60 and $125 \mathrm{~d}$ of age, animals in RE group received a quantity of feed limited to $75-80 \%$ of their estimated needs based on weight, whereas animals from AL group were fed ad libitum. Subsequently, all animals were fed ad libitum for a period of 30-35 d, until $\sim 5$ months of age (161 $\pm 0.5 \mathrm{~d}$ of age; $96.8 \pm 1.8 \mathrm{~kg}$ of live weight), when they were slaughtered. Pigs were weighed individually at the beginning and every 2 weeks during the whole experiment (at the age of $8,10,12,14,16,18,20$ and 22 weeks), plus the day before slaughtering. Additionally, back fat thickness (BFT) in vivo was measured with a PIGLOG 105 ultrasound equipment in the third ( $30 \mathrm{~kg}$ of live weight) and successive controls (i.e. at 12, 14, 16, 18, 20 and 22 weeks of age approximately). Individual feed intake during the two periods of the trial (feed restriction and realimentation/CG periods) was also recorded for all RE and $\mathrm{AL}$ gilts. Individual growth and feed conversion ratio during each period were computed.

Animals were slaughtered at IRTA Experimental Slaughterhouse in Monells (Girona, Spain) in totally controlled conditions and in compliance with all welfare regulations. Before slaughter, all RE and AL sows were fasted for $12 \mathrm{~h}$ and stunned with high concentrations of $\mathrm{CO}_{2}$ before bleeding. All experimental procedures were approved by the Ethical Committee of IRTA.

\section{Phenotypic data}

After slaughtering, carcass weight and AutoFOM2 measures of lean percentage, loin thickness and BFT between the third and fourth ribs were registered at the slaughterhouse. Moreover, samples of $\sim 100 \mathrm{~g}$ of gluteus medius (GM) muscle were collected for laboratory analyses. Electric conductivity (EC), ultimate $\mathrm{pH}\left(\mathrm{pH}_{24}\right)$, and meat colour parameters (lightness $\mathrm{L}^{*}$, redness $\mathrm{a}^{*}$ and yellowness $b^{*}$ ) were determined 24-h after slaughtering following the methods described in Gonzalez-Prendes et al. [14]. Analyses of GM lipid components included the determination of percentage of intramuscular fat (IMF), cholesterol content and fatty acid composition in the C12 - C22 interval, as described in Canovas et al. [15]. Statistical differences between group means were assessed with a two-tailed t-test. 
In order to perform RNA-Seq analysis, additional GM muscle samples were collected immediately after slaughter and submerged in RNAlater (Sigma, Spain) before storage at $-80{ }^{\circ} \mathrm{C}$, according to the protocols recommended by the manufacturer.

\section{RNA extraction, library construction and sequencing}

The GM samples were individually ground with a mortar and a pestle to homogenization on liquid nitrogen. The RNA of the samples was extracted using the Ambion RiboPure (Thermo Fisher Scientific). Total RNA was quantified in a Nanodrop ND-1000 spectrophotometer and RNA purity and integrity was checked by using a Bioanalyzer- 2100 equipment (Agilent Technologies, INC., Santa Clara, CA). Libraries were prepared using the TruSeq RNA Sample Preparation Kit (Ilumina Inc., CA). Pools of three libraries per line with barcoding were paired-end sequenced $(2 \times$ 75 bp), by using the TruSeq SBS Kit v3-HS (Illumina Inc., CA), in a HiSeq 2000 platform (Illumina Inc., CA). All sequencing tasks were carried out in the Centro Nacional de Análisis Genómico (Barcelona, Spain).

\section{Mapping, annotation and differential expression analysis}

The quality of the raw sequenced reads in the FASTQ files was analysed with the FASTQC software (Babraham Bioinformatics, http://www.bioinformatics.babraham.ac.uk/projects/fastqc/). Reads were mapped to the reference pig genome Sscrofa10.2 and the annotation database Ensembl Genes 86 (http://www.ensembl.org/info/data/ftp/index.html) by using STAR v. 2.5.2a [16]. Mapping quality evaluation and descriptive statistics were assessed with Qualimap v. 2.2. [17]. The number of reads mapping to each gene were obtained with the HTSeq-count tool included in the HTSeq python library [18] by using the same GTF file of the alignment step. The R package DESeq2 [19] was used to identify differentially expressed (DE) genes based on the RNA-seq data. Genes with a Fold Change (FC) above 2 (i.e. $\left.\mid \log _{2} \mathrm{FCl}>1\right)$ and significant $P$-value after correcting for multiple testing $\left(P_{\text {adj }}\right.$-value $\left.<0.05\right)$ were classified as DE. Gene ontologies (GO), metabolic pathways, and biological functions significantly $\left(p_{\text {adj }}<0.05\right)$ enriched in the set of DE genes were determined using the ClueGO v. 2.3.5 plug-in of Cytoscape v. 3.2.1 [20] and the Core Analysis function included in the Ingenuity Pathway Analysis software (IPA; Ingenuity Systems). For functional analyses, orthologous human gene names were retrieved from the Ensembl Genes 89 Database using the Biomart software [21].

\section{Results}

\section{Phenotypic differences}

Phenotypic means of RE and AL groups for production and meat quality traits are presented in Table 1. Our results show that RE animals had significantly lower growth rates than the $\mathrm{AL}$ ones during the restriction period but they experienced compensatory growth when they were fed ad libitum, thus surpassing daily gains obtained in AL group ( $1.14 \mathrm{~kg}$ in RE vs $0.97 \mathrm{~kg}$ in AL; $P$-value $<0.005)$. Growth curves for both RE and AL animals are shown in Fig. 1. After $\sim 35 \mathrm{~d}$ of re-feeding, the "compensatory index value" in our animals was $26 \%$ (calculated as reviewed in [13]). In addition, no significant differences in feed intake were observed between groups when both RE and AL gilts were fed ad libitum. Such observation is explained by the better feed conversion rate of RE gilts during CG (2.53 vs $2.93 \mathrm{~g}$ intake / g gain in RE and AL groups respectively; $P$-value $<0.001$ ).

Despite undergoing a phase of accelerated growth, RE animals did not reach the same live weight than AL animals at slaughter age (150-160 d). This way, large differences were observed between RE and AL groups with regard to live weight, RE animals weighed about $12 \mathrm{~kg}$ less than AL animals at slaughter time. Such gap reduced to $10 \mathrm{~kg}$ of difference in carcass weights, and a suggestively $(P$-value $=0.064)$ lower killing out percentage was observed in RE animals $(79.03 \%$ vs $80.03 \%$ in RE vs AL groups; Table 1).

As far as fatness is concerned, Duroc females fed a restricted diet tended to deposit more subcutaneous fat during the subsequent CG period than their AL counterparts (6.21 vs $5.00 \mathrm{~mm}$ of back fat gain at CG period in RE vs AL group; $P$-value $<0.1)$. However, at the age of slaughter RE gilts still showed lower back fat thickness than AL ones $(22.28 \mathrm{~mm}$ in $\mathrm{RE}$ vs $25.62 \mathrm{~mm}$ in $\mathrm{AL}$; $P$-value $<0.05)$ and a suggestive $(P$-value $<0.1)$ gain in the percentage of lean meat. Consistently with these observations, our results point out that RE animals also tend to have a lower percentage of IMF content than the AL ones $(P$-value $<0.1)$, at least during the CG following the restriction period.

Regarding IMF composition of the GM muscle, important differences were also observed in the muscle fatty acid profile of the two groups of animals. The most remarkable difference was the higher polyunsaturated fatty acids (PUFA) content showed by RE gilts in the CG period when compared to the AL ones $(13.82 \%$ vs $11.35 \%$ of PUFA in RE vs AL pigs). Consistently, the PUFA/SFA ratio was higher in RE sows. The higher PUFA content in RE animals during CG is mainly due to an increased amount of omega- 6 fatty acids in the GM of RE sows $(10.83 \%$ in $\mathrm{RE}$ vs $8.85 \%$ in $\mathrm{AL}$ ). The content of linoleic acid, the shortest-chained omega- 6 fatty acid, and to a lesser extent the arachidonic acid content, were the main causes for these differences. Omega-3 PUFA content was also higher in the RE group, but differences did not become significant. Finally, it is worth mentioning that animals subjected to a period of food restriction tended to have a lower percentage of monounsaturated fatty acids (MUFA), including palmitoleic and oleic acids, at the end of the subsequent CG period. 
Table 1 Mean phenotypic values for growth, fatness, carcass and intramuscular fat traits of the two groups of pig females analysed, fed ad libitum (AL group) and fed a restricted diet (RE group), plus the significance of dietary treatment effect in a one way ANOVA

\begin{tabular}{|c|c|c|c|}
\hline & $\mathrm{AL}$ & RE & $P$-value ${ }^{a}$ \\
\hline \multicolumn{4}{|l|}{ GROWTH from 70 to $125 \mathrm{~d}$ of age (restriction period) } \\
\hline Average daily gain (kg) & 0.88 & 0.55 & $<.0001 * * *$ \\
\hline Daily feed intake (kg) & 2.05 & 1.26 & $<.0001 * * *$ \\
\hline Food conversion ratio (kg intake / kg gain) & 2.33 & 2.27 & 0.2863 n.s. \\
\hline Back fat gain from 85 to $125 \mathrm{~d}(\mathrm{~mm})$ & 5.25 & 2.75 & $<.0001 * * *$ \\
\hline \multicolumn{4}{|l|}{ GROWTH from 126 to $155 \mathrm{~d}$ of age (re-feeding period) } \\
\hline Average daily gain (kg) & 0.97 & 1.14 & $0.0046 * *$ \\
\hline Daily feed intake (kg) & 2.84 & 2.88 & 0.8060 n.s. \\
\hline Food conversion ratio (kg intake / kg gain) & 2.93 & 2.53 & $0.0005 * * *$ \\
\hline Back fat gain $(\mathrm{mm})$ & 5.00 & 6.21 & $0.0569+$ \\
\hline \multicolumn{4}{|l|}{ CARCASS TRAITS ( 161 d of age) } \\
\hline Live weight before slaughter (kg) & 101.10 & 89.10 & $<.0001 * * *$ \\
\hline Killing out (\%) & 80.03 & 79.03 & $0.0640+$ \\
\hline Chilling losses (\%) & 3.01 & 3.59 & $0.0015 * *$ \\
\hline Back fat thickness between 3rd and 4th ribs (Fat-O-Meater II) (mm) & 25.52 & 22.28 & $0.0243 *$ \\
\hline Loin thickness between 3rd and 4th ribs (Fat-O-Meater II) (mm) & 44.13 & 40.30 & $0.0239 *$ \\
\hline Lean meat percentage (FOM 2) & 50.16 & 52.33 & $0.0851+$ \\
\hline \multicolumn{4}{|l|}{ INTRAMUSCULAR FAT TRAITS (gluteus medius) } \\
\hline Intramuscular fat content (\%) & 3.733 & 3.120 & $0.0542+$ \\
\hline Cholesterol content (dg/kg) & 58.082 & 58.193 & 0.9695 n.s. \\
\hline Myristic acid (C14:0) content (\%) & 1.348 & 1.291 & 0.3641 n.s. \\
\hline Palmitic acid (C16:0) content (\%) & 23.844 & 23.326 & 0.1258 n.s. \\
\hline Palmitoleic acid (C16:1n7) content (\%) & 3.003 & 2.662 & $0.0164 *$ \\
\hline Stearic acid (C18:0) content (\%) & 13.713 & 13.926 & 0.6280 n.s. \\
\hline Oleic acid (C18:1n9) content (\%) & 40.100 & 38.454 & $0.0806+$ \\
\hline Cis-vaccenic acid (C18:1n7) content (\%) & 3.960 & 3.853 & 0.1893 n.s. \\
\hline Linoleic acid (C18:2n6) content (\%) & 8.531 & 10.185 & $0.0110 *$ \\
\hline Arachidonic acid (C20:4n6) content (\%) & 1.440 & 1.874 & $0.0630+$ \\
\hline Saturated Fatty Acids content (SFA) (\%) & 39.403 & 38.991 & 0.5857 n.s. \\
\hline Monounsaturated fatty acids content (MUFA) (\%) & 49.249 & 47.156 & $0.0529+$ \\
\hline Polyunsaturated fatty acids content (PUFA) (\%) & 11.347 & 13.818 & $0.0149 *$ \\
\hline Ratio PUFA/SFA & 0.288 & 0.356 & $0.0158 *$ \\
\hline Omega $6(\omega 6)$ content & 8.851 & 10.832 & $0.0089 * *$ \\
\hline Omega $3(\omega 3)$ content & 0.933 & 1.270 & 0.1762 n.s. \\
\hline Ratio $\omega 6 / \omega 3$ & 9.935 & 9.816 & 0.9016 n.s. \\
\hline Sum of trans FA & 0.804 & 0.799 & 0.9678 n.s. \\
\hline
\end{tabular}

${ }^{\mathrm{a} S i g n i f i c a n t: ~}{ }^{* * *} p<0.0001 ;{ }^{* *} p<0.01 ;{ }^{*} p<0.05$; Suggestive: $+p<0.1$; n.s.: non-significant

Conversely to IMF fatty acids profile, no differences in $\mathrm{pH}$, EC nor muscle colour parameters $\left(\mathrm{L}^{*}, \mathrm{a}^{*}\right.$ and $\left.\mathrm{b}^{*}\right)$ were observed between RE and AL animals (data not shown).

\section{Transcriptome analysis of the gluteus medius muscle} The sequencing the $24 \mathrm{GM}$ muscle samples resulted in a total of $2430 \mathrm{M}$ of $75 \mathrm{bp}$ paired-end reads. A general description about the total reads mapped in the sequencing process is reported in Additional file 2: Table S2. A total of $89.6 \%$ (from 88.3 to $91.6 \%$ ) of reads were mapped to the porcine reference genome Sscrofa10.2, and approximately $17.4 \%$ (from 14.7 to $19.0 \%$ ) of them mapped at more than one location. Of the total mapped reads, $70.6 \%(66.4-73.6 \%)$ corresponded to annotated 


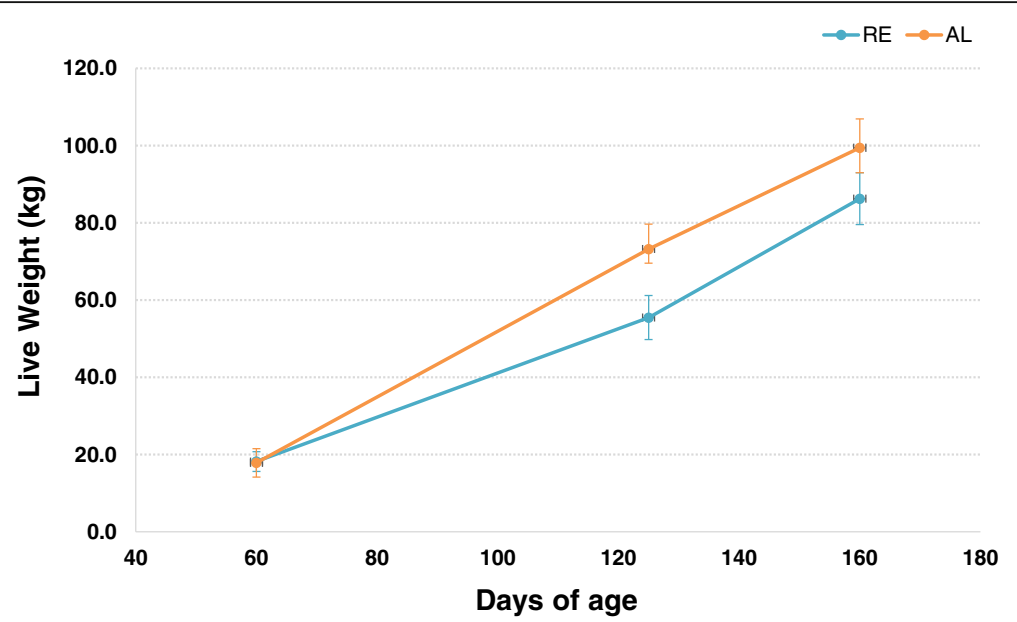

Fig. 1 Evolution of live weight of animals subjected to feed restriction from 70 to $125 \mathrm{~d}$ of age (RE animals) and fed ad libitum (AL animals) across the controlled fattening period till sacrifice ( $155 \mathrm{~d}$ of age)

genes, $80.1 \%(77.8-83.2 \%)$ were located in exonic regions, and $8.2 \%$ in intronic regions (7.0-9.7\%). Finally, $11.7 \%(9.2-17.7 \%)$ of the reads were mapped in intergenic regions.

\section{Differentially expressed genes}

A multidimensional scaling (MDS) plot based on the log-fold-changes between each pair of RNA samples (Fig. 2) shows that biological replicates from the same group (RE and $\mathrm{AL}$ ) still cluster together even $35 \mathrm{~d}$ after delivering the same nutritional treatment to $\mathrm{RE}$ and $\mathrm{AL}$ sows. This plot indicates the existence of clear differences in the muscle transcriptomic profile of these two groups of animals. However, some samples of RE and AL groups are more similar to samples of different treatments indicating that the refeeding starts to dilute the differences between both groups (Fig. 2).

Differential expression analysis using DESeq2 allowed identifying a total of 86 genes $\mathrm{DE}\left(\log _{2} \mathrm{FCl}>1, P_{\text {adj-value }}\right.$ $<0.05$ ) between RE and AL sows (Additional file 3: Table S3). Most of these DE genes, 73 out of 86 , showed higher expression levels in AL sows, whereas only 13 genes showed higher expression in RE sows. If we apply a slightly less restrictive threshold regarding $\mathrm{FC}$, for example $\mid \log _{2} \mathrm{FCl}>0.85$, the number of genes differentially expressed between groups increases to

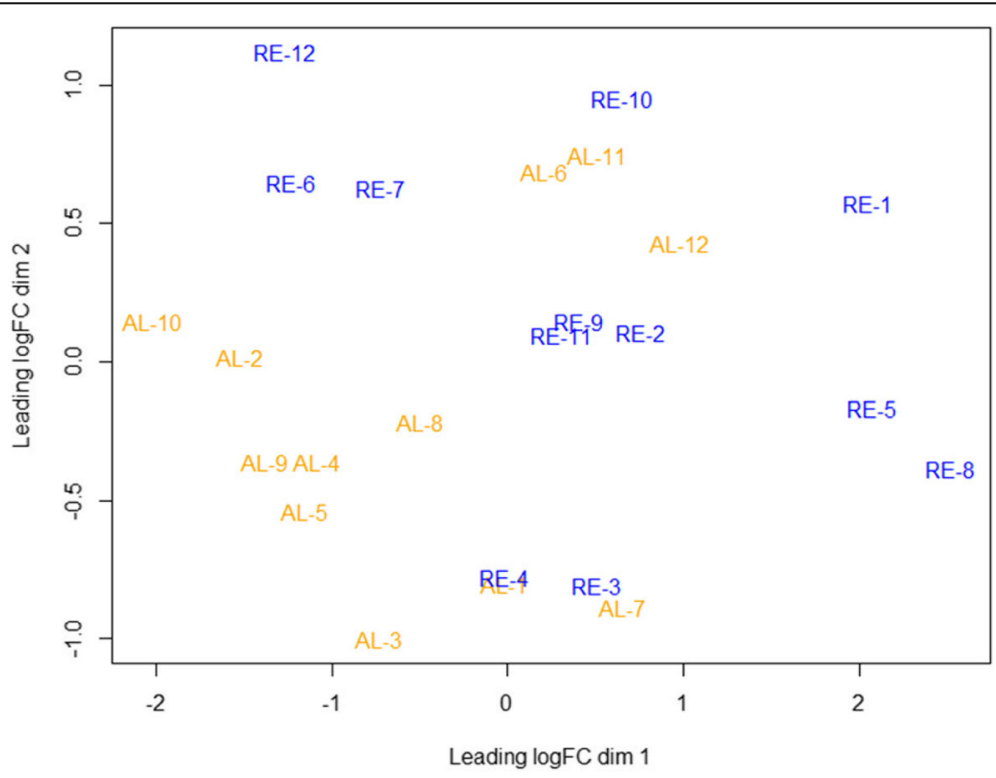

Fig. 2 Multidimensional scaling (MDS) plot based on the log-fold-changes between each pair of RNA samples. Orange color indicates AL sows and blue color indicates RE sows 
214. However, the majority of $\mathrm{DE}$ genes remain to be overexpressed in AL sows: 162 genes upregulated and 52 genes down-regulated in the AL group when compared to the RE group (Additional file 3: Table S3). Prior to the functional analysis of DE genes, a detailed look of Additional file 3: Table S3 revealed that the list of the most significant DE genes contains a number of loci involved in energy metabolism and autophagy, e.g. PFKFB3, MKL1, PPARGC1A, PRKAG2, NR4A3 or DUSP4.

\section{Functional analysis}

To perform a functional classification of genes showing differential expression between RE and AL groups, a subset of 72 annotated genes having an orthologous human gene (out of the 86 genes considered as DE with $\mathrm{FC}>2$ in Additional file 3: Table S3) was submitted to IPA. The six canonical pathways most significantly $(P$-value $<0.001)$ enriched in the list of DE genes is shown in Table 2. These genes are involved in G-protein coupled receptor signaling, AMPK signaling, relaxin signaling, ERK/MAPK signaling, phagosome formation and type II diabetes mellitus signaling. The remaining pathways identified in the list of $\mathrm{DE}$ genes are shown in Additional file 4: Table S4. It is worth to highlight PPAR $\alpha / R X R \alpha$ activation, NRF2-mediated oxidative stress response, TR/RXR activation, IGF-1 signaling, insulin receptor signaling and triacylglycerol degradation among the canonical pathways identified.

Additionally, a gene ontology analysis to identify the biological processes enriched in the set of DE genes was performed by using ClueGO. AMPK signaling and adipocytokine signalling were two of the most overrepresented KEGG pathways, and energy homeostasis was the biological process most significantly enriched in the set of $\mathrm{DE}$ genes. Furthermore, the regulation of lipid metabolism by PPAR alpha was also overrepresented. This way, overall results indicate that regulation of energy homeostasis plays a key role in the muscle transcriptome differences observed between RE animals and their AL counterparts during the CG process. Remarkably, the Z-score for the AMPK signaling pathway was negative (-1.633; Table 2), indicating molecules participating in this process have the same directional effect. Indeed, almost all DE genes taking part in this pathway (PIK3R1, PRKAG2, FRS2, PFKFB2, and PPARGC1A) were down-regulated in the RE group compared to the AL one (Additional file 3: Table S3).

The most significant networks identified by IPA in the list of DE genes are listed in Table 3. Among the top five networks, three of them were associated to either carbohydrate metabolism, cardiovascular system, cell cycle and survival, or lipid metabolism, whilst the other two networks were associated to several diseases or metabolic disorders. The most significantly enriched network, mainly associated with carbohydrate metabolism, is presented in Fig. 3. Finally, among the principal functions most represented in the aforementioned identified networks (Additional file 5: Table S5), it is worth mentioning the uptake of D-glucose, glycolysis, oxidation of fatty acid, lipolysis, density of mitochondria, quantity of mitochondrial DNA, synthesis of ATP, production of reactive oxygen species, and apoptosis, among others.

\section{Discussion}

Despite several endocrine and metabolic studies have been conducted in several species, there is still a lack of information about the mechanisms implicated in CG in pigs. The analysis performed in the current study about the changes in the skeletal muscle transcriptome may improve our understanding about the biological and molecular mechanisms underlying CG induced by re-alimentation after a feed restriction period.

\section{Compensatory growth and its biological factors}

In the present study, RE animals showed a CG index of $26 \%$. This value indicates an incomplete CG, as the CG index generally ranges between 50 and 100\% [13]. As previously mentioned, our experiment was designed to analyse changes in the transcriptome occurring during the CG period, and animals were slaughtered after $\sim 35 \mathrm{~d}$ of re-feeding, so before completing the standard fattening and CG period to reach the commercial weight (around $120 \mathrm{~kg}$ of live weight in the studied Duroc population). This way, RE pigs showed lower live and carcass weights when compared to AL ones. Also viscera organ weight was $1.48 \mathrm{~kg}$ lower, but represented $1 \%$

Table 2 Top six canonical pathways most significantly ( $P$-value $<0.001)$ enriched by genes differentially expressed between RE and AL groups

\begin{tabular}{lllll}
\hline Canonical Pathway & $-\log _{10}(p$-value $)$ & Ratio & z-score & Molecules $^{a}$ \\
\hline G-Protein Coupled Receptor Signaling & $4.53 \mathrm{E} 00$ & $2.57 \mathrm{E}-02$ & - & SMPDL3A, OPRD1, PDE7B, PIK3R1, PRKAG2, DUSP4, FRS2 \\
AMPK Signaling & $4.45 \mathrm{E} 00$ & $3.17 \mathrm{E}-02$ & -1.633 & PFKFB3, PIK3R1, PRKAG2, FRS2, PFKFB2, PPARGC1A \\
Relaxin Signaling & $3.85 \mathrm{E} 00$ & $3.29 \mathrm{E}-02$ & - & SMPDL3A, PDE7B, PIK3R1, PRKAG2, FRS2 \\
ERK/MAPK Signaling & $3.31 \mathrm{E} 00$ & $2.51 \mathrm{E}-02$ & -1.342 & PLA2G4A, PIK3R1, PRKAG2, DUSP4, FRS2 \\
Phagosome Formation & $3.16 \mathrm{E} 00$ & $3.28 \mathrm{E}-02$ & - & MRC1, PIK3R1, TLR1, FRS2 \\
Type II Diabetes Mellitus Signaling & $3.10 \mathrm{E}+00$ & $3.15 \mathrm{E}-02$ & -1.000 & PIK3R1, CD36, PRKAG2, FRS2 \\
\hline
\end{tabular}

${ }^{\mathrm{a}}$ The bold italic-lettered genes were those down-regulated in the RE group relative to the AL group 
Table 3 Top five networks enriched by genes differentially expressed between RE and AL groups. Principal functions represented in those biological networks are indicated in Additional file 5: Table S5

\begin{tabular}{lll}
\hline Associated Network functions & Score & Molecules \\
\hline Carbohydrate Metabolism, Cardiovascular System Development and Function, Cellular Development & 44 & 20 \\
Cell Cycle, Cell Death and Survival, Glomerular Injury & 28 & 14 \\
Cancer, Organismal Injury and Abnormalities, Renal and Urological Disease & 28 & 14 \\
Lipid Metabolism, Small Molecule Biochemistry, Carbohydrate Metabolism & 23 & 12 \\
Auditory Disease, Hereditary Disorder, Neurological Disease & 23 & 12 \\
\hline
\end{tabular}

more of live weight in RE vs AL pigs. In the first weeks following feed intake restriction, a relatively low basal metabolism has been observed due to the reduced weight of viscera in RE animals [13] which need some time to recover the capacity of the gastrointestinal tract [8]. Thus, during this first period of CG it is usual to observe increased live weight gains up to $20 \%$ [7].

Among the biological factors accounting for CG, changes in feed intake and feed efficiency have been reported [7, 8]. In our study, RE animals did not present an increase in feed intake after the restriction period but they showed a better feed conversion ratio during the CG phase when compared with their AL counterparts (Table 1). After feed restriction, growing pigs need about $3-4$ weeks to recover the capacity and size of the gastrointestinal tract and for this reason the feed intake of those pigs increases gradually [8]. Similar results were observed in heifers when considering the whole realimentation period [22]. The better feed efficiency in RE animals might be due to a slow increase of basal metabolism as well as to lower maintenance requirements and the more efficient use of protein and energy during the beginning of realimentation [22].

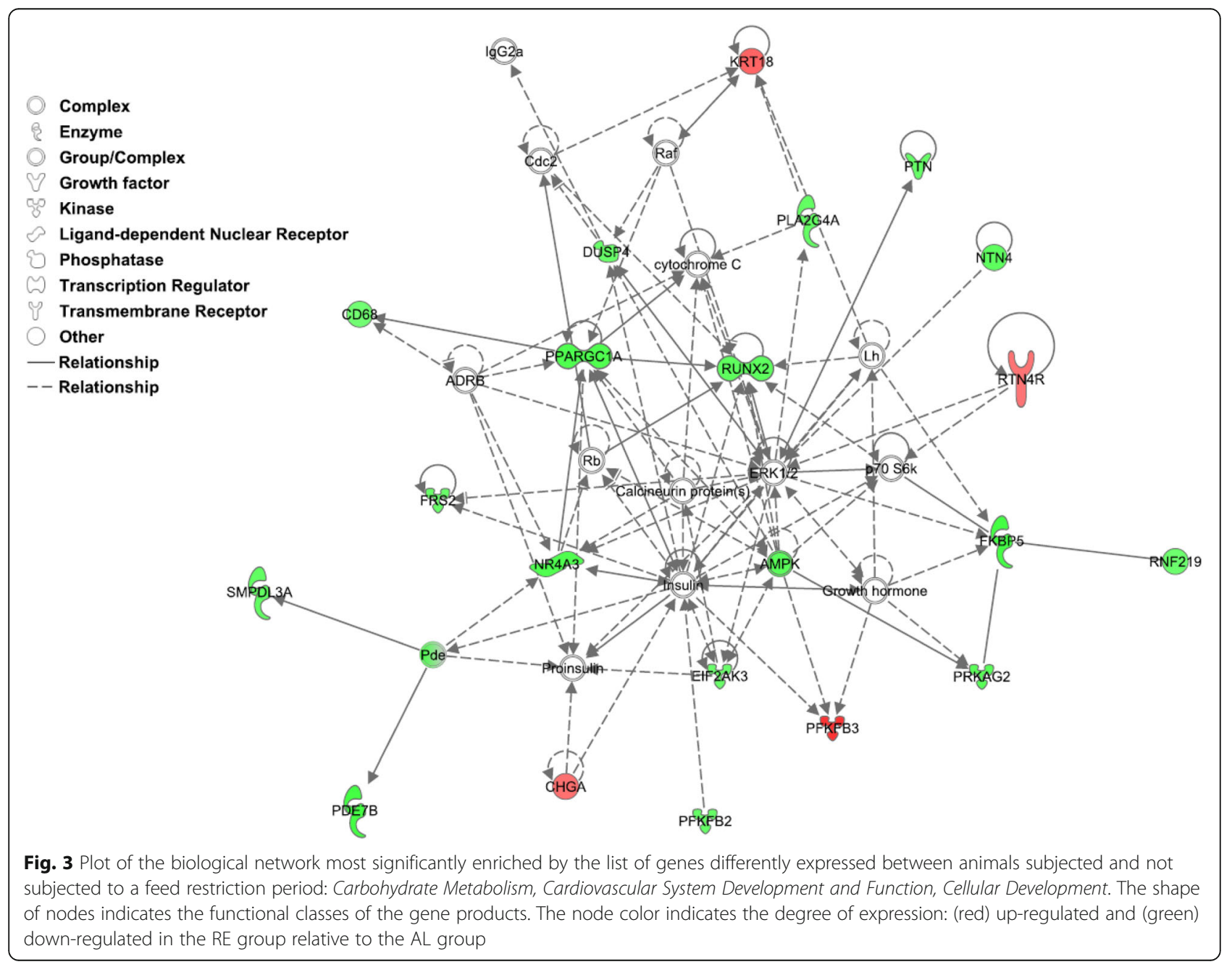


Overall, we can assume that our transcriptome data offer a picture of what happens at the molecular level during the beginning of the CG period. However, we cannot discard other factors affecting the CG recovery of our animals as it has previously been described that this phenomenon varies depending on multiple factors such as the type, degree, timing and duration of growth restriction among others [7]. In the next sections, we will discuss the biological pathways and processes identified as overrepresented in the list of genes DE between RE and AL sows, thus aiming to decipher the molecular mechanisms contributing to CG and changes in muscle composition.

\section{Energy production and maintenance requirements}

A main finding at the functional level was the down-regulation of the AMPK pathway and its downstream catabolic processes in animals that had been subjected to feed restriction (RE group). AMPK acts as a key sensor of the AMP/ATP ratio, stimulating the activation of catabolic processes to generate ATP (reviewed in [23]). In our study, the protein kinase AMP-activated non-catalytic subunit gamma 2 (PRKAG2) showed much higher expression levels in $\mathrm{AL}$ animals compared with the RE ones (FC $=2.62, P$-value $\left.=8.45 \times 10^{-8}\right)$. The gamma subunits of AMPK are important, as they interact with AMP and ATP molecules detecting directly the cellular levels of ATP, ADP and AMP [24].

One of the immediate consequences of AMPK activation is an increase in glucose uptake and decrease in glycogen synthesis rated, coupled to an increase of glycolysis in skeletal muscle [23]. In our study, the top network identified by IPA was associated with carbohydrate metabolism (Fig. 3), whose overrepresented functions include the uptake of monosaccharide, uptake of D-glucose, metabolism of fructose-2, 6-diphosphate and glycolysis. DE genes gathered in this network were mainly up-regulated in AL group when compared with RE one (Additional file 5: Table S5). Among the set of genes under-expressed in RE animals, it is worthy to highlight the Nuclear Receptor Subfamily 4 Group A Member $3\left(N R 4 A 3 ; \mathrm{FC}=2.44, P\right.$-value $\left.=1.03 \times 10^{-5}\right)$ and 6-Phosphofructo-2-Kinase/Fructose-2, 6-Biphosphatase 2 (PFKFB 2; $\quad \mathrm{FC}=2.19, \quad P$-value $\left.=2.85 \times 10^{-7}\right)$ genes. The NR4A3 gene regulates the translocation of the SLC2A4 glucose transporter (also called GLUT4) to the surface of skeletal muscle cells to increase the uptake of glucose [25], while PFKFB2 catalyses the synthesis of fructose-2, 6-bisphosphate, a key regulator of glycolysis [26].

AMPK also stimulates fatty acid oxidation and mitochondrial biogenesis to increase ATP levels through the activation of PPARG Coactivator 1 Alpha (PPARGC1A; also known as $P G C-1 \alpha)$ in the muscle [23, 27]. PGC-1 $\alpha$ is a transcriptional co-activator that, among others transcription factors, activates PPARs to induce mitochondrial gene expression and promote oxidative metabolism
[28]. In our study, PPARGC1A was also upregulated in the $\mathrm{AL}$ group compared with the $\mathrm{RE}$ one $(\mathrm{FC}=2.44, P$-value $=$ $5.52 \times 10^{-5}$ ). This agrees well with the upregulation in the AL group of genes involved in both PPAR $\alpha / R X R \alpha$ Activation canonical pathway and oxidation of lipid function (Additional file 3: Table S3 and Additional file 4: Table S4). In addition, we found the density of mitochondria, quantity of mitochondrial DNA, and synthesis of ATP among the overrepresented functions identified (Additional file 5: Table S5). Remarkably, while an increase of mitochondrial biogenesis to provide ATP has been described in animals under calorie restriction [29], a metabolic shift to less energy production was observed in cattle undergoing CG after restriction, with downregulation of genes involved in oxidative phosphorylation and the citric acid cycle [12]. Hence, Keogh et al. [12] indicated that mitochondrial energy production efficiency is not a relevant process contributing to the compensation of skeletal muscle tissue and overall CG in re-fed animals after restriction. This conclusion is in close agreement with the down-regulation of the AMPK pathway observed in our RE animals during the subsequent CG period. In addition, coupled with this result, we observed a decrease ( $\mathrm{z}$-score $=-2216$ ) in the expression of genes involved in production of reactive oxygen species (ROS) in the RE group. Interestingly, a decrease in ROS production has also been associated to pigs showing better feed efficiency (low residual feed intake) in several studies $[30,31]$. This result agrees with the improvement in the food conversion ratio of animals undergoing CG reported in our study (Table 1). Furthermore, the NRF2-mediated Oxidative Stress Response was among the canonical pathways identified by us as having DE genes downregulated in $\mathrm{RE}$ vs AL animals. Another mechanism to limit the production of ROS is through the autophagy degradation of mitochondria [32].

Finally, among genes upregulated in animals subjected to feed restriction, the 6-Phosphofructo-2-Kinase/Fructose-2, 6-Biphosphatase 3 gene (PFKFB3) resulted to be the most upregulated gene in $\mathrm{RE}$ vs $\mathrm{AL}$ animals $(\mathrm{FC}=2.80, P$-value $\left.=5.26 \times 10^{-7}\right)$. This gene as its paralog, the PFKFB2 gene cited above, controls glycolysis and it has been also associated with cell proliferation and prevention of apoptosis [33]. In fact, upregulation of this gene has been described to be implicated in the Warburg effect in cancer cells to fulfil their high energy demands [34]. As a matter of fact, PFKFB 3 but not PFKFB1, PFKFB2 nor PFKFB4 has been identified as a novel downstream substrate of mTOR signalling pathway [35]. The mTOR pathway is essential in cell metabolism, controlling protein biosynthetic processes, growth and proliferation. This pathway, which was among the canonical pathways identified (Additional file 4: Table S4), is blocked by AMPK [23]. Interestingly, this result is in agreement with the elevated glycolytic potential observed by Gondret et al. [36] in the muscle of pigs selected for feed 
efficiency (low residual feed intake), in opposition to the more fatty acid oxidative profile presented by less efficient (high residual feed intake) animals. In our study, in addition to genes associated with fatty acids oxidation, also genes related with triacylglycerol degradation (ABHD2 and LIPG) were identified as DE, all of them upregulated in the $\mathrm{AL}$ group compared with the $\mathrm{RE}$ one (Additional file 4: Table S4).

Altogether these results suggest that animals subjected to a feed restriction period have more glycolytic potential and less oxidative metabolic profile and catabolic processes in skeletal muscle during the subsequent CG period than animals fed ad libitum. Overall these changes may be coupled with the higher feed efficiency observed in restricted animals at the beginning of CG due to the reduced maintenance requirements.

\section{Skeletal muscle autophagy is downregulated during compensatory growth}

Besides its role as a key sensor of the AMP/ATP ratio, the AMPK pathway plays a fundamental role in the regulation of autophagy [37], a biological process involving the degradation of cell components and molecules that are engulfed in autophagosomes that subsequently are fused with lysosomes [38]. Under stress conditions, such as dietary restriction, the recycling of macromolecules mediated by autophagy provides energy-rich compounds that can be used to restore metabolic homeostasis. From yeast to mammals, the strong activation of autophagy in response to food deprivation has been convincingly demonstrated to be a fundamental mechanism to supply nutrients to starved cells [39]. Moreover, there is evidence that autophagy plays an essential role in maintaining muscle mass and myofiber integrity [38], and that excessive activation of autophagy in the skeletal muscle (e.g. during starvation) induces a severe loss of muscular mass [40].

The downregulation of PRKAG2, which encodes a subunit of AMPK, in RE pigs would suggest that muscle autophagy is reduced during CG. Indeed, AMPK is a central activator of autophagy by inactivating TORC1 and phosphorylating $U L K 1[37,41]$. In addition, we have identified six genes (STX3, FKBP5, ACSL1, RUNX2, DUSP4 and $E I F 2 A K 3$ ) that are downregulated in RE pigs (Additional file 3: Table S3) and that also activate autophagy. For instance, the Runt related transcription factor 2 (RUNX2) gene facilitates autophagy in metastatic cancer breast cells by increasing acetylation of $\alpha$-tubulin sub-units of microtubules [42], and autophagic cell death in head and neck squamous cell carcinoma is mediated by DUSP4 (Dual Specificity Phosphatase 4) [43]. The knockdown of the Acyl-CoA Synthetase Long chain family member 1 (ACSL1) gene impairs cardiac autophagy [44], and the Eukaryotic Translation Initiation Factor 2 Alpha Kinase 3 (EIF2AK3) gene is a crucial mediator of endoplasmic reticulum stress-induced autophagy [45]. The implication of FK506 binding protein 5 (FKBP5) gene in promoting autophagy has been also reported [46], and Syntaxin 3 (STX3) gene is involved in vesicle trafficking and fusion [47].

On the other hand, in close support with our view that autophagy is decreased during CG, we have detected three genes (KDM2B, MKL1 and PFKFB3) that inhibit autophagy and are upregulated in RE pigs (Additional file 3: Table S3). The downregulation of $K D M 2 B$ (Lysine-specific demethylase 2B) in gastric cancer cells immediately induces autophagy followed by an inhibition of cell proliferation [48], and the inactivation of Megakaryoblastic Leukemia (Translocation) 1 (MKL1) gene in mouse embryos causes myocardial cell necrosis that could be the consequence of a decreased ability of the myocardium to cope with environmental stresses [49]. The case of the PFKFB3 gene, the most upregulated gene in RE vs AL animals, is even more enlightening. The product of this gene controls the conversion of fructose-6-phosphate to and from fructose-2, 6-bisphosphate, a key regulator of the glycolytic enzyme phosphofructokinase-1 [50]. The inhibition of PFKFB3 has been shown to decrease glucose uptake and to promote autophagy in cancer cells [50]. Indeed, glucose deprivation is known to activate autophagy via AMPK while mTORC1 would be concomitantly inhibited [51]. Moreover, the mTOR signalling pathway, for which PFKFB3 has been identified as a downstream substrate, is also a key inhibitor of autophagy in response to nutritional status, growth factor and stress signals [52].

\section{Cellular growth and function}

As stated before, PFKFB3 has been also associated with cell proliferation and prevention of apoptosis. In the RE group, the second most upregulated gene compared with AL group was the $M K L 1$ gene $(\mathrm{FC}=2.40, P$-value $=1.10 \times$ $\left.10^{-8}\right)$. MKL1 is a member of the myocardin-related transcription factor (MRTF) family which regulates a wide variety of essential biological processes such as muscle cell differentiation, cell survival and apoptosis [53]. More recently, MKL1 has been involved in the control of global transcriptional activity and cellular growth through regulating chromatin structure [54]. Although in our study genes related with anti-apoptotic function, cell proliferation and cell growth have been identified as upregulated in restricted animals, there is not an overrepresentation of cellular growth and proliferation functions in the list of genes DE between RE and AL groups.

\section{Fat depot and intramuscular fatty acid composition}

During the restriction period, restricted gilts displayed a lower back fat gain when compared with ad libitum fed animals. In the re-alimentation period, the CG response is mainly focused to restore internal organ growth and body fat stores, although initially CG is characterized by 
an increased protein accretion $[1,8,13]$. In our study, RE animals also showed an increased back fat gain during CG period when compared with $\mathrm{AL}$ ones, but the refeeding period ( $\sim 35 \mathrm{~d}$ ) was not long enough to bridge the gap between RE and AL animals regarding fat deposition and IMF content. Similar results have been observed in pigs refed during $28 \mathrm{~d}$ or $34 \mathrm{~d}$ after restriction; therefore, it was concluded that meat eating quality was not improved in pigs under CG and that IMF content might be modulated by modifying the duration of the restriction and refeeding periods (reviewed in [1]).

Besides differences in the IMF content, also variations in the muscular fatty acids profile of sows subjected to a feed restriction period and CG were also observed in our study. While RE sows presented higher levels of PUFA content than the AL ones, and more particularly of omega- 6 fatty acids (mainly linoleic acid but also arachidonic), the MUFA content, including oleic and palmitoleic acids, tended to be lower than those of AL sows. Consistently Daza et al. [2] described a higher concentration of essential fatty acids (such as linoleic acid) and a lower concentration of non-essential fatty acids in the back fat tissue of Iberian pigs subjected to severe feed restriction. These authors explained such results on the basis of a reduced activity of lipogenic enzymes. However, we did not identify lipogenic genes differentially expressed between RE and $\mathrm{AL}$ groups in our study. Conversely to that, RE animals presented an increase in the expression of genes related with glycolysis, cellular growth and prevention of apoptosis. We could hypothesize that our RE animals were in an initial phase of CG, characterized by the deposition of lean tissue, and therefore back fat content and IMF composition are still a reflection of the feed restriction period.

In our study, RE pigs showed an increased back fat gain in the $\sim 35 \mathrm{~d}$ of re-feeding, but they were slaughtered before completing CG and reaching the commercial weight and age, so they did neither reach the point of maximum fat deposition. This circumstance prevents us from drawing conclusions about the pork composition of restricted animals in commercial fattening conditions after a longer re-feeding period.

\section{Conclusions}

We propose that food restriction and CG constitute two contrary metabolic poles, while ad libitum feeding would represent a third intermediate condition. Our study focuses on changes in the pig skeletal muscle transcriptome profile during the phase of accelerated growth in the initial CG period after feed restriction. We have identified genes, biological processes and gene networks that may be implicated at the molecular level in the physiological mechanism of CG, and may play a role in the variations observed on IMF fatty acid profile. RE animals presented a more glycolytic potential, and less oxidative metabolic profile in muscle than AL animals. Overall these changes may be coupled with the lower food conversion ratio observed in RE animals due to the reduced maintenance requirements observed at the beginning of realimentation and CG. Our results also indicate that CG may induce a downregulation of autophagy in the skeletal muscle of pigs as a mechanism to increase muscle mass.

These results provide a new perspective about the molecular basis of CG in a monogastric species, revealing that autophagy could be an important player in pig growth. Further studies are needed to determine if AMPK pathway remains as the main mechanism underlying changes in muscle transcriptome in later phases of CG.

\section{Additional files}

Additional file 1: Table S1. Composition of the fed used. (XLSX $10 \mathrm{~kb}$ )

Additional file 2: Table S2. Description of reads mapped in the RNASeq procedure for the 24 animals analysed. (DOCX $12 \mathrm{~kb}$ )

Additional file 3: Table S3. List of genes differentially expressed ( $p$-value adjusted for multiple testing $<0.05$ ) between animals subjected and not subjected to a feed restriction period, considering two additional thresholds regarding Fold Change (FC): a) $\mathrm{FC}>2$ (i.e. $\log _{2} \mathrm{FCl}>1$ ) and b) $\mathrm{FC}>1.8$ (i.e. $\left.\log _{2} \mathrm{FCl}>0.85\right)$. (XLSX $38 \mathrm{~kb}$ )

Additional file 4: Table S4. List of canonical pathways identified at the list of 86 genes differentially expressed $\left(P_{\text {adj }}\right.$-value $<0.05$ and $\log _{2} \mathrm{FCl}>1$ ) between animals subjected and not subjected to a feed restriction period. (XLS 64 kb)

Additional file 5: Table S5. Principal functions represented in the biological networks identified in the list of differently expressed between animals subjected and not subjected to feed restriction. (XLS 149 kb)

\section{Abbreviations}

a: Redness; ABHD2: Abhydrolase domain containing 2; ACSL1: Acyl-CoA synthetase long chain family member 1; ADP: Adenosine diphosphate; AL: fed ad libitum; AMP: Adenosine monophosphate; AMPK: AMP-activated protein kinase; ATP: Adenosine triphosphate; b: Yellowness; BFT: Back fat thickness; CG: Compensatory growth; DE: Differentially expressed; DNA: Deoxyribonucleic acid; DUSP4: Dual specificity phosphatase 4; EC: Electric conductivity; EIF2AK3: Eukaryotic translation initiation factor 2 alpha kinase 3; FA: Fatty acids; FC: Fold Change; FKBP5: FK506 binding protein 5; FRS2: Fibroblast growth factor receptor substrate 2; GM: Gluteus medius muscle; GO: Gene ontology; IGF-1: Insulin-like growth factor 1; IMF: Intramuscular fat; IPA: Ingenuity Pathway Analysis software; KDM2B: Lysine demethylase 2B; L: Lightness; LIPG: Lipase G;

MDS: Multidimensional scaling; MKL1: Megakaryoblastic Leukemia (Translocation) 1 gene; mTORC1: Mammalian target of rapamycin complex 1; MUFA: Monounsaturated fatty acids; NR4A3: Nuclear receptor subfamily 4 group A member 3; NRF2: Nuclear factor, erythroid 2 like 2; PFKFB1: 6phosphofructo-2-kinase/fructose-2, 6-biphosphatase 1; PFKFB2: 6phosphofructo-2-kinase/fructose-2, 6-biphosphatase 2; PFKFB3: 6phosphofructo-2-kinase/fructose-2, 6-biphosphatase 3; PFKFB4: 6phosphofructo-2-kinase/fructose-2, 6-biphosphatase 4; $\mathrm{pH}$ 24: Ultimate $\mathrm{pH}$; PIK3R1: Phosphoinositide-3-kinase regulatory subunit 1; PPARGC1A: PPARG coactivator 1 alpha; PPARs: Peroxisome proliferator-activated receptors; PPARa/RXRa: Peroxisome proliferator-activated receptor alpha/Retinoid $X$ receptor alpha; PRKAG2: Protein kinase AMP-activated non-catalytic subunit gamma 2; PUFA: Polyunsaturated fatty acids; RE: fed under restriction; RNA: Ribonucleic acid; RNA-Seq: RNA sequencing; ROS: Reactive oxygen species; RUNX2: Runt related transcription factor 2; SFA: Saturated fatty acids; SLC2A4 (GLUT4): Solute carrier family 2 member 4; STX3: Syntaxin 3;

TORC1: Target of rapamycin complex 1 kinase; TR/RXR: Thyroid hormone receptor/heterodimers with retinoid $X$ receptor; ULK1: Unc-51 like autophagy activating kinase 1; w3: Omega 3; w6: Omega 6 


\section{Acknowledgements}

Authors are indebted to Selección Batallé S.A. for providing the animal material. We gratefully acknowledge to J. Reixach (Selección Batallé), J. Soler (IRTA), C. Millan (IRTA), A. Quintana (IRTA) and A. Rossell (IRTA) for their collaboration in the experimental protocols at farm and slaughterhouse.

\section{Funding}

The study was funded by grants AGL2013-48742-C2-1-R and AGL201348742-C2-2-R, awarded by the Spanish Ministry of Economy and Competitivity (MINECO). We also acknowledge the support of the Spanish Ministry of Economy and Competitivity for the Center of Excellence Severo Ochoa 2016-2019 (SEV-2015-0533) grant awarded to the Centre for Research in Agricultural Genomics (CRAG). Maria Ballester is financially supported by a Ramon y Cajal contract (RYC-2013-12573) from the Spanish Ministry of Economy and Competitiveness. Tainã Figueiredo Cardoso was funded with a fellowship from the CAPES Foundation-Coordination of Improvement of Higher Education, Ministry of Education of the Federal Government of Brazil. Rayner Gonzalez-Prendes was funded with a FPU Ph.D. grant from the Spanish Ministry of Education (FPU12/00860). Thanks also to the CERCA Programme of the Generalitat de Catalunya. The funders had no role in study design, data collection and analysis, decision to publish or preparation of the manuscript.

\section{Availability of data and materials}

Sequence data have been deposited in the NCBI Sequence Read Archive (SRA) database under BioProject number PRJNA386796.

\section{Authors' contributions}

$\mathrm{RQ}$ and MA designed the experiment; $\mathrm{RQ}$ and $\mathrm{JT}$ were responsible for the experimental device and production phenotypes; NPR and ID performed carcass measurements and meat laboratory analyses; all authors contributed to the obtaining of biological samples; TFC and RGP performed RNA extraction and quality control; MB, MP and OGR analysed the RNA-Seq data; MB performed differential expression and functional analyses; MB, MA and $\mathrm{RQ}$ wrote the paper. All authors read and approved the manuscript.

\section{Ethics approval}

Animal care, management procedures and tissue sampling were performed following national guidelines for the Good Experimental Practices and they were approved by the Ethical Committee of the Institut de Recerca i Tecnologia Agroalimentàries (IRTA). The study was carried out with commercial Duroc animals from Batallé (http://www.batalle.com).

\section{Consent for publication}

Not applicable.

\section{Competing interests}

The authors declare that they have no competing interests.

\section{Publisher's Note}

Springer Nature remains neutral with regard to jurisdictional claims in published maps and institutional affiliations.

\footnotetext{
Author details

${ }^{1}$ Animal Breeding and Genetics Programme, Institute for Research and Technology in Food and Agriculture (IRTA), Torre Marimon, E08140 Caldes de Montbui, Spain. ${ }^{2}$ Department of Animal Genetics, Center for Research in Agricultural Genomics (CSIC-IRTA-UAB-UB), Universitat Autònoma de Barcelona, E08193 Bellaterra, Spain. ${ }^{3}$ Product Quality Programme, Institute for Research and Technology in Food and Agriculture (IRTA), Finca Camps i Armet, E17121 Monells, Spain. ${ }^{4}$ Animal Breeding and Genetics Programme, Institute for Research and Technology in Food and Agriculture (IRTA), Finca Camps i Armet, E17121 Monells, Spain.
}

Received: 28 February 2018 Accepted: 11 September 2018 Published online: 17 September 2018

\section{References}

1. Lebret B. Effects of feeding and rearing systems on growth, carcass composition and meat quality in pigs. Animal. 2008;2:1548-58.
2. Daza A, Mateos A, Rey Al, Ovejero I, López-Bote CJ. Effect of duration of feeding under free-range conditions on production results and carcass and fat quality in Iberian pigs. Meat Sci. 2007;76:411-6.

3. Kristensen L, Therkildsen M, Riis B, Sørensen MT, Oksbjerg N, Purslow PP, et al. Dietary-induced changes of muscle growth rate in pigs: effects on in vivo and postmortem muscle proteolysis and meat quality. J Anim Sci. 2002;80:2862-71.

4. Therkildsen M, Riis B, Karlsson A, Kristensen L, Ertbjerg P, Purslow PP, et al. Compensatory growth response in pigs, muscle protein turn-over and meat texture: effects of restriction/realimentation period. Anim Sci. 2002;75:367-77.

5. Kristensen L, Therkildsen M, Aaslyng MD, Oksbjerg N, Ertbjerg P, Compensatory growth improves meat tenderness in gilts but not in barrows. J Anim Sci. 2004:82:3617-24.

6. Therkildsen M, Vestergaard M, Busk H, Jensen MT, Riis B, Karlsson AH, et al Compensatory growth in slaughter pigs-in vitro muscle protein turnover at slaughter, circulating IGF-I, performance and carcass quality. Livest Prod Sci. 2004;88:63-75.

7. Martínez-Ramírez H, de Lange C. Compensatory Growth in Pigs. Recent Adv Anim. Nutr. 2007. p. 331-52.

8. Skiba G. Physiological aspects of compensatory growth in pigs. J Anim Feed Sci. 2005:14:191-203.

9. Wood JD, Richardson RI, Nute GR, Fisher AV, Campo MM, Kasapidou E, et al. Effects of fatty acids on meat quality: a review. Meat Sci. 2004:21-32.

10. Wood JD, Enser M, Fisher a V, Nute GR, Sheard PR, Richardson RI, et al. Fat deposition, fatty acid composition and meat quality: a review. Meat Sci. 2008;78:343-58.

11. Heyer A, Lebret B. Compensatory growth response in pigs: effects on growth performance, composition of weight gain at carcass and muscle levels, and meat quality. J Anim Sci. 2007:85:769-78.

12. Keogh K, Kenny DA, Cormican P, McCabe MS, Kelly AK, Waters SM. Effect of dietary restriction and subsequent re-alimentation on the transcriptional profile of bovine skeletal muscle. PLoS One. 2016:11:e0149373.

13. Hornick JL, Van Eenaeme C, Gérard O, Dufrasne I, Istasse L. Mechanisms of reduced and compensatory growth. Domest Anim Endocrinol. 2000;19:121-32.

14. González-Prendes R, Quintanilla R, Cánovas A, Manunza A, Figueiredo Cardoso T, Jordana J, et al. Joint QTL mapping and gene expression analysis identify positional candidate genes influencing pork quality traits. Sci Rep. 2017:7:39830.

15. Cánovas A, Quintanilla R, Amills M, Pena RN. Muscle transcriptomic profiles in pigs with divergent phenotypes for fatness traits. BMC Genomics. 2010; 11:372.

16. Dobin A, Gingeras TR. Mapping RNA-seq reads with STAR. Curr Protoc Bioinforma. 2015:51:11.14.1-19.

17. Okonechnikov K, Conesa A, García-Alcalde F. Qualimap 2: advanced multisample quality control for high-throughput sequencing data. Bioinformatics. 2016;32:292-4.

18. Anders S, Pyl PT, Huber W. HTSeq--a Python framework to work with highthroughput sequencing data. Bioinformatics. 2015;31:166-9.

19. Love Ml, Huber W, Anders S. Moderated estimation of fold change and dispersion for RNA-seq data with DESeq2. Genome Biol. 2014;15:550.

20. Bindea G, Mlecnik B, Hackl H, Charoentong P, Tosolini M, Kirilovsky A, et al. ClueGO: a Cytoscape plug-in to decipher functionally grouped gene ontology and pathway annotation networks. Bioinformatics. 2009;25:1091-3.

21. Smedley D, Haider S, Durinck S, Pandini L, Provero P, Allen J, et al. The BioMart community portal: an innovative alternative to large, centralized data repositories. Nucleic Acids Res. 2015;43:W589-98.

22. Yambayamba ES, Price MA, Foxcroft GR. Hormonal status, metabolic changes, and resting metabolic rate in beef heifers undergoing compensatory growth. J Anim Sci. 1996;74:57-69.

23. Cantó C, Auwerx J. AMP-activated protein kinase and its downstream transcriptional pathways. Cell Mol Life Sci. 2010;67:3407-23.

24. Xiao B, Heath R, Saiu P, Leiper FC, Leone P, Jing C, et al. Structural basis for AMP binding to mammalian AMP-activated protein kinase. Nature. 2007:449:496-500.

25. Liu Q, Zhu X, Xu L, Fu Y, Garvey WT. 6-Mercaptopurine augments glucose transport activity in skeletal muscle cells in part via a mechanism dependent upon orphan nuclear receptor NR4A3. Am J Physiol Endocrinol Metab. 2013;305:E1081-92.

26. Heine-Suñer D, Díaz-Guillén MA, Lange AJ, Rodríquez de Córdoba S. Sequence and structure of the human 6-phosphofructo-2-kinase/ fructose-2,6-bisphosphatase heart isoform gene (PFKFB2). Eur J Biochem. 1998;254:103-10. 
27. Cantó C, Auwerx J. Calorie restriction: is AMPK a key sensor and effector? Physiology (Bethesda). 2011;26:214-24.

28. Vega RB, Huss JM, Kelly DP. The coactivator PGC-1 cooperates with peroxisome proliferator-activated receptor alpha in transcriptional control of nuclear genes encoding mitochondrial fatty acid oxidation enzymes. Mol Cell Biol. 2000;20:1868-76.

29. López-Lluch G, Hunt N, Jones B, Zhu M, Jamieson H, Hilmer S, et al. Calorie restriction induces mitochondrial biogenesis and bioenergetic efficiency. Proc Natl Acad Sci U S A. 2006;103:1768-73.

30. Grubbs JK, Fritchen AN, Huff-Lonergan E, Dekkers JCM, Gabler NK, Lonergan SM. Divergent genetic selection for residual feed intake impacts mitochondria reactive oxygen species production in pigs. J Anim Sci. 2013;91:2133-40.

31. Ramayo-Caldas Y, Ballester M, Sánchez JP, González-Rodríguez O, Revilla M, Reyer $\mathrm{H}$, et al. Integrative approach using liver and duodenum RNA-Seq data identifies candidate genes and pathways associated with feed efficiency in pigs. Sci Rep. 2018;8:558.

32. Glick D, Barth S, Macleod KF. Autophagy: cellular and molecular mechanisms. J Pathol. 2010;221:3-12.

33. Moncada S, Higgs EA, Colombo SL. Fulfilling the metabolic requirements for cell proliferation. Biochem J. 2012;446:1-7.

34. Almeida A, Bolaños JP, Moncada S. E3 ubiquitin ligase APC/C-Cdh1 accounts for the Warburg effect by linking glycolysis to cell proliferation. Proc Natl Acad Sci U S A. 2010;107:738-41.

35. Feng Y, Wu L. mTOR up-regulation of PFKFB3 is essential for acute myeloid leukemia cell survival. Biochem Biophys Res Commun. 2017:483:897-903.

36. Gondret F, Vincent A, Houée-Bigot M, Siegel A, Lagarrigue S, Causeur D, et al. A transcriptome multi-tissue analysis identifies biological pathways and genes associated with variations in feed efficiency of growing pigs. BMC Genomics. 2017;18:244

37. Kim J, Kundu M, Viollet B, Guan K-L. AMPK and mTOR regulate autophagy through direct phosphorylation of Ulk1. Nat Cell Biol. 2011;13:132-41.

38. Fiacco E, Castagnetti F, Bianconi V, Madaro L, De Bardi M, Nazio F, et al. Autophagy regulates satellite cell ability to regenerate normal and dystrophic muscles. Cell Death Differ. 2016;23:1839-49.

39. Shang L, Chen S, Du F, Li S, Zhao L, Wang X. Nutrient starvation elicits an acute autophagic response mediated by Ulk1 dephosphorylation and its subsequent dissociation from AMPK. Proc Natl Acad Sci U S A. 2011;108:4788-93.

40. Masiero E, Agatea L, Mammucari C, Blaauw B, Loro E, Komatsu M, et al. Autophagy is required to maintain muscle mass. Cell Metab. 2009;10:507-15.

41. Hardie DG. AMPK and autophagy get connected. EMBO J. 2011;30:634-5.

42. Tandon M, Othman AH, Ashok V, Stein GS, Pratap J. The role of Runx2 in facilitating autophagy in metastatic breast cancer cells. J Cell Physiol. 2018;233:559-71.

43. Li K-C, Hua K-T, Lin Y-S, Su C-Y, Ko J-Y, Hsiao M, et al. Inhibition of G9a induces DUSP4-dependent autophagic cell death in head and neck squamous cell carcinoma. Mol Cancer. 2014;13:172.

44. Grevengoed TJ, Cooper DE, Young PA, Ellis JM, Coleman RA. Loss of longchain acyl-CoA synthetase isoform 1 impairs cardiac autophagy and mitochondrial structure through mechanistic target of rapamycin complex 1 activation. FASEB J. 2015;29:4641-53.

45. Zhao C, Yin S, Dong Y, Guo X, Fan L, Ye M, et al. Autophagydependent EIF2AK3 activation compromises ursolic acid-induced apoptosis through upregulation of MCL1 in MCF-7 human breast cancer cells. Autophagy. 2013;9:196-207.

46. Gassen NC, Hartmann J, Schmidt MV, Rein T. FKBP5/FKBP51 enhances autophagy to synergize with antidepressant action. Autophagy. 2015;11:578-80.

47. Soo Hoo L, Banna CD, Radeke CM, Sharma N, Albertolle ME, Low SH, et al. The SNARE protein Syntaxin 3 confers specificity for polarized axonal trafficking in neurons. PLoS One. 2016;11:e0163671.

48. Zhao E, Tang C, Jiang X, Weng X, Zhong X, Zhang D, et al. Inhibition of cell proliferation and induction of autophagy by KDM2B/FBXL10 knockdown in gastric cancer cells. Cell Signal. 2017;36:222-9.

49. Sun Y, Boyd K, Xu W, Ma J, Jackson CW, Fu A, et al. Acute myeloid leukemia-associated Mkl1 (Mrtf-a) is a key regulator of mammary gland function. Mol Cell Biol. 2006;26:5809-26.

50. Klarer AC, O'Neal J, Imbert-Fernandez Y, Clem A, Ellis SR, Clark J, et al. Inhibition of 6-phosphofructo-2-kinase (PFKFB3) induces autophagy as a survival mechanism. Cancer Metab. 2014;2:2.

51. Moruno F, Pérez-Jiménez E, Knecht E. Regulation of autophagy by glucose in mammalian cells. Cell. 2012;1:372-95.
52. Jung $\mathrm{CH}$, Ro S-H, Cao J, Otto NM, Kim D-H. mTOR regulation of autophagy. FEBS Lett. 2010;584:1287-95.

53. Scharenberg MA, Chiquet-Ehrismann R, Asparuhova MB. Megakaryoblastic leukemia protein-1 (MKL1): increasing evidence for an involvement in cancer progression and metastasis. Int J Biochem Cell Biol. 2010;42:1911-4.

54. Flouriot G, Huet G, Demay F, Pakdel F, Boujrad N, Michel D. The actin/MKL1 signalling pathway influences cell growth and gene expression through large-scale chromatin reorganization and histone post-translational modifications. Biochem J. 2014;461:257-68.

\section{Ready to submit your research? Choose BMC and benefit from:}

- fast, convenient online submission

- thorough peer review by experienced researchers in your field

- rapid publication on acceptance

- support for research data, including large and complex data types

- gold Open Access which fosters wider collaboration and increased citations

- maximum visibility for your research: over $100 \mathrm{M}$ website views per year

At BMC, research is always in progress.

Learn more biomedcentral.com/submissions 